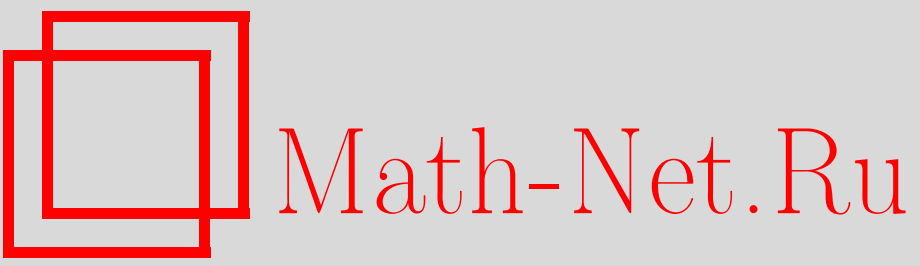

С. П. Зубова, Е. В. Раецкая, Л. Чунг, О сохранении траектории при возмущениях в линейной динамической системе управления, Итоги науки и техн. Сер. Соврем. мат. и ее прил. Темат. обз., 2021, том 190, 93-106

DOI: https://doi.org/10.36535/0233-6723-2021-190-93-106

Использование Общероссийского математического портала Math-Net.Ru подразумевает, что вы прочитали и согласны с пользовательским соглашением

http://www.mathnet.ru/rus/agreement

Параметры загрузки:

IP : 52.205 .19 .152

26 апреля 2023 г., 10:59:17 


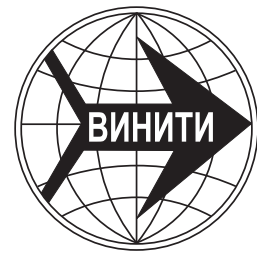

ИТОГИ НАУКИ И ТЕХНИКИ.

Современная математика и ее приложения.

Тематические обзоры.

Том 190 (2021). С. 93-106

DOI: 10.36535/0233-6723-2021-190-93-106

УДК 517.518

\title{
О СОХРАНЕНИИ ТРАЕКТОРИИ ПРИ ВОЗМУЩЕНИЯХ В ЛИНЕЙНОЙ ДИНАМИЧЕСКОЙ СИСТЕМЕ УПРАВЛЕНИЯ
}

\author{
(c) 2021 г. $\quad$ С. П. ЗУБОВА, Е. В. РАЕЦКАЯ, ЛЕ ХАЙ ЧУНГ
}

\begin{abstract}
АннотАция. Для линейной нестационарной полностью управляемой динамической системы с многоточечными условиями на состояние ставится задача независимости состояния (траектории) системы от внешних возмущений и возможных изменений параметров системы (внутренних возмущений). Задача состоит в построении управления для возмущенной системы, при реализации которого состояние возмущенной системы идентично состоянию невозмущенной системы. Производится сравнение управляющих воздействий для невозмущенной и возмущенной систем при одинаковых состояниях систем.
\end{abstract}

Ключевъе слова: линейная динамическая система, управление, возмущение, инвариантность, блокировка, метод каскадной декомпозиции.

\section{ON THE INVARIANCE OF TRAJECTORIES UNDER PERTURBATIONS IN LINEAR DYNAMIC CONTROL SYSTEMS}

\author{
(C) 2021 S. P. ZUBOVA, E. V. RAETSKAYA, LE HAY TRUNG
}

\begin{abstract}
For a linear, nonstationary, completely controllable dynamical system with multipoint conditions on the state, we consider a problem on the independence of the state (trajectory) of the system of external perturbations and possible changes in the parameters of the system (internal perturbations). The problem is to construct a control for the perturbed system under which the state of the perturbed system is identical to the state of the unperturbed system. We compare controls for the unperturbed and perturbed systems at the same states of the systems.
\end{abstract}

Keywords and phrases: linear dynamical system, control, perturbation, invariance, blocking, cascade decomposition method.

AMS Subject Classification: $34 \mathrm{H} 05$

1. Введение. Защита динамического процесса от помех, шумов, возмущений, а также сохранение состояния системы при измененных параметрах динамической системы является одной из важнейших задач автоматического управления.

В частности, актуальна следующая задача: при появлении возмущений, изменений параметров системы изменить управляющее воздействие так, чтобы состояние системы осталось бы неизменным, таким же, как и до появления возмущений. То есть сделать систему жесткой, инвариантной, не реагирующей на помехи, шумы, возмущения с помощью определенного управления.

В [8] отмечено свойство гашения некоторого возмущения $H$ с помощью $D$ в линейной стационарной динамической системе

$$
\dot{x}_{v}(t)=(B+D H) x_{v}(t)+D u_{v}(t)
$$

Работа выполнена при поддержке Фонда развития науки и техники Министерства образования и обучения Вьетнама (проект № B2017.DNA.09). 
в том смысле, что эта система является полностью управляемой, если невозмущенная система

$$
\dot{x}(t)=B x(t)+D u(t)
$$

полностью управляема.

Это свойство гашения возмущений с помощью оператора $D$ используется, в частности, в настоящей статье для сохранения программной траектории динамической системы с широким спектром возмущений.

Рассматривается линейная неоднородная система управления с переменными коэффициентами и с различными возмущениями. Показывается, что многоточечная задача управления разрешима или неразрешима одновременно для невозмущенной и возмущенной определенным образом систем. Для возмущенной системы строится управляющее воздействие такое, что состояние ее такое же, как состояние невозмущенной системы, удовлетворяющее многоточечным условиям. Производится сравнение управляющих воздействий для возмущенной и невозмущенной систем с целью установить, насколько следует изменить управление при появлении возмущений, чтобы динамическая система была инвариантной по отношению к возмущениям. Рассматривается программное управление, возмущения или их определенные «части», считаются известными.

2. Постановка задачи и предварительные сведения. Пусть динамическая система

$$
\dot{x}(t)=B(t) x(t)+D(t) u(t)+f(t),
$$

где $x(t) \in \mathbb{R}^{n}, u(t) \in \mathbb{R}^{m}, f(t) \in \mathbb{R}^{n} ; B(t)$ и $D(t)$ - матрицы соответствующих размеров; $t \in$ $\left[t_{0}, t_{k}\right] ; B(t), D(t)$ и $f(t)$ непрерывны на $\left[t_{0}, t_{k}\right]$, полностью управляема. Здесь $u(t)$ - управляющая вектор-функция, под влиянием которой вектор-функция состояния системы $x(t)$ переходит из произвольного начального состояния $x\left(t_{0}\right)=x_{0}$ в произвольное конечное состояние $x\left(t_{k}\right)=x_{k}$, проходя через любые наперед заданные точки $x\left(t_{i}\right)=x_{i}, i=1,2, \ldots, k-1, t_{0}<t_{1}<\ldots<t_{k}$, т.е.

$$
x\left(t_{i}\right)=x_{i}, \quad i=0,1,2, \ldots, k .
$$

Пусть в системе (1) возникли шумы, помехи, некоторые изменения и т. д. и возмущенная система описывается уравнением

$$
\left(I+H_{1}(t)\right) \dot{x}_{v}(t)=\left(B(t)+H_{2}(t)\right) x_{v}(t)+\left(D(t)+H_{3}(t)\right) u_{v}(t)+f(t)+h(t)
$$

с непрерывными матрицами $H_{i}(t), i=1, \ldots, 3$, и непрерывной вектор-функцией $h(t)$.

Задача состоит в построении такой непрерывной вектор-функции $u_{v}(t)$, что вектор-функция состояния $x_{v}(t)$ системы $(3)$ тождественна вектор-функции состояния $x(t)$ в задаче $(1),(2)$ :

$$
x_{v}(t) \equiv x(t), \quad t \in\left[t_{0}, t_{k}\right]
$$

Следует также установить, насколько отличается $u_{v}(t)$ от $u(t)$ в зависимости от величин возмущающих факторов, т.е. как и насколько следует изменить управление возмущенной системой, чтобы состояние системы не реагировало на возмущения.

Для решения поставленных задач применяется метод каскадной декомпозиции задач (каскадный метод), разработанный, в частности, и для решения задач управления (см. [2, 4, 5]), опирающийся на следующее свойство (отображения и соответствующие им матрицы обозначаются одинаково).

Если $C(t): \mathbb{R}^{s} \rightarrow \mathbb{R}^{l}$, то имеют место при каждом $t \in\left[t_{0}, t_{k}\right]$ разложения $\mathbb{R}^{s}$ и $\mathbb{R}^{l}$ в прямые суммы

$$
\mathbb{R}^{s}=\operatorname{Coim} C(t) \dot{+} \operatorname{Ker} C(t), \quad \mathbb{R}^{l}=\operatorname{Im} C(t) \dot{+} \operatorname{Coker} C(t),
$$

где $\operatorname{Ker} C(t)$ - нуль-пространство (ядро) отображения $C(t) ; \operatorname{Im} C(t)$ - множество значений $C(t)$, Coker $C(t)$ - дефектное подпространство, Coim $C(t)$ - прямое дополнение к $\operatorname{Ker} C(t)$ в $\mathbb{R}^{s}$.

Введем следующие обозначения: $P(C(t))$ и $Q(\underset{\widetilde{C}}{C(t))}$ - проекторы на $\operatorname{Ker} C(t)$ и $\operatorname{Coker} C(t)$ соответственно, отвечающие этим разложениям; $\widetilde{C}(t)$ - сужение $C(t)$ на $\operatorname{Coim} C(t), C^{-}(t)=$ $\widetilde{C}(t)^{-1}(I-Q(C(t)))$ - полуобратное отображение, $I$ - тождественное отображение в соответствующем пространстве, далее это единичные матрицы. При этом $P(C(t))=I-C^{-}(t) C(t)$ и $Q(C(t))=I-C(t) C^{-}(t)$.

Если при некотором значении $t$ матрица $C(t)$ нулевая, то полагаем $C^{-}(t)$ - нулевая матрица. 
Отображение $C(t)$ называют сюръективным, если его множество значений совпадает с $\mathbb{R}^{l}$, т.е. если $Q(C(t)) \equiv 0$.

Далее для краткости символ $(t)$ в проекторах опускается.

Каскадный метод основан на расщеплении уравнения вида $C(t) v(t)=w(t)$ на уравнения в подпространствах $\operatorname{Im} C(t)$ и Coker $C(t)$ и применения следующей леммы.

Лемма 1 (см. [2]). Соотношение $C(t) v(t)=w(t), v(t) \in \mathbb{R}^{s}, w(t) \in \mathbb{R}^{l}$, эквивалентно системе

$$
\left\{\begin{array}{l}
Q(C) w(t)=0 \\
v(t)=C^{-}(t) w(t)+z(t)
\end{array}\right.
$$

где $z(t)=P(C) v(t)-$ произвольный элемент из $\operatorname{Ker} C(t)$.

Заметим, что разложения пространств в прямые суммы могут быть записаны в разных формах, проекторы $P(C), Q(C)$ и полуобратное отображение $C^{-}(t)$ также могут иметь разные формы записи, но результаты леммы 1 при разных формах записи являются эквивалентными. К примеру, если

то система

$$
C=\left(\begin{array}{ll}
1 & 1 \\
1 & 1
\end{array}\right)
$$

$$
\left\{\begin{array}{l}
v_{1}+v_{2}=w_{1} \\
v_{1}+v_{2}=w_{2}
\end{array}\right.
$$

эквивалентна одной из систем

$$
\left\{\begin{array} { l } 
{ w _ { 1 } - w _ { 2 } = 0 } \\
{ ( \begin{array} { l } 
{ v _ { 1 } } \\
{ v _ { 2 } }
\end{array} ) = ( \begin{array} { c } 
{ w _ { 1 } } \\
{ 0 }
\end{array} ) + ( \begin{array} { r } 
{ - v _ { 2 } } \\
{ v _ { 2 } }
\end{array} ) }
\end{array} \quad \text { или } \quad \left\{\begin{array}{l}
-w_{1}+w_{2}=0, \\
\left(\begin{array}{l}
v_{1} \\
v_{2}
\end{array}\right)=\left(\begin{array}{c}
0 \\
w_{2}
\end{array}\right)+\left(\begin{array}{r}
v_{1} \\
-v_{1}
\end{array}\right) .
\end{array}\right.\right.
$$

Здесь

$$
\begin{aligned}
& P(C)=\left(\begin{array}{rr}
0 & -1 \\
0 & 1
\end{array}\right) \quad \text { или } P(C)=\left(\begin{array}{rr}
1 & 0 \\
-1 & 0
\end{array}\right) ; \\
& Q(C)=\left(\begin{array}{rr}
1 & -1 \\
0 & 0
\end{array}\right) \quad \text { или } Q(C)=\left(\begin{array}{rr}
0 & 0 \\
-1 & 1
\end{array}\right) \text {, } \\
& C^{-}=\left(\begin{array}{ll}
1 & 0 \\
0 & 0
\end{array}\right) \quad \text { или } \quad C^{-}=\left(\begin{array}{ll}
0 & 0 \\
0 & 1
\end{array}\right) .
\end{aligned}
$$

Пусть $D(t)$ несюръективен при всех $t \in\left[t_{0}, t_{k}\right]$. Применение леммы 1 к уравнению $(1)$, записанному в виде

$$
D(t) u(t)=\dot{x}(t)-B(t) x(t)-f(t),
$$

согласно лемме при $C(t)=D(t)$ приводит к формуле для нахождения $u(t)$ в виде функциональной зависимости от $x(t)$ и к уравнению для нахождения $x(t)$; это уравнение в свою очередь преобразуется к виду системы управлений с некоторыми функциями псевдосостояния и псевдоуправления. $\mathrm{K}$ этой новой системе управления снова применяется лемма 1 и т. д. Этот процесс изложен пошагово в [4] в двух вариантах: с помощью проекторов на подпространства и с помощью линейных замен искомых функций.

Применение леммы к уравнению (3), записанному в виде

$$
D(t) u_{v}(t)=-H_{3}(t) u_{v}(t)+\left(I+H_{1}(t)\right) \dot{x}_{v}(t)-\left(B(t)+H_{2}(t)\right) x_{v}(t)-f(t)-h(t),
$$

также приводит к формуле для нахождения $u_{v}(t)$ через $x_{v}(t)$ и к уравнению для нахождения $x_{v}(t)$.

Поставленная выше задача разрешима за счет того, что уравнения для нахождения $x(t)$ и $x_{v}(t)$ при определенных свойствах возмущений одинаковы.

Рассматриваются два случая: когда возмущающие операторы $H_{i}(t), i=1, \ldots, 3$, сюръективны и $h(t) \in \operatorname{Im} D(t)$, и случай возмущений общего вида. В случае произвольных возмущений рассматривается возможность блокировки возмущений введением в систему блокатора, роль которого выполняет $D(t)$. 


\section{3. Решение невозмущенной задачи.}

3.1. Первый шаг декомпозииии. Предлагается решение задачи (1), (2) методом каскадной декомпозиции (см. $[2,4,5])$.

Согласно лемме 1 уравнение (5), т.е. (1), эквивалентно системе

$$
\begin{aligned}
u(t) & =D^{-}(t)(\dot{x}(t)-B(t) x(t)-f(t))+z(t), \\
Q(D) \dot{x}(t) & \equiv Q(D)(B(t) x(t)+f(t)),
\end{aligned}
$$

с произвольной вектор-функцией $z(t) \in \operatorname{Ker} D(t)$. Соотношение $(8)$ - это условие корректности системы (1).

Равенство (7) есть формула для определения $u(t)$ после того, как из (8) будет найдено $x(t)$, удовлетворяющее условиям (2). Решение задачи (8), (2) можно осуществить следующим образом.

В результате дифференцирования $Q(D)$ и замены

$$
\left\{\begin{array}{l}
Q(D) x(t)=x^{1}(t), \\
(I-Q(D)) x(t)=y^{1}(t)
\end{array}\right.
$$

уравнение (8) записывается в виде

$$
\dot{x}^{1}(t)=B_{1}(t) x^{1}(t)+D_{1}(t) y^{1}(t)+f_{1}(t)
$$

где

$$
\begin{gathered}
B_{1}(t)=(\dot{Q}(D)+Q(D) B(t)) Q(D), \\
D_{1}(t)=(\dot{Q}(D)+Q(D) B(t))(I-Q(D)), \\
f_{1}(t)=Q(D) f(t) .
\end{gathered}
$$

В представлении (10) использованы свойства проекторов $Q(D)$ и $(I-Q(D))$ :

$$
Q^{2}(D)=Q(D), \quad(I-Q(D))^{2}=(I-Q(D)),
$$

в силу чего

$x^{1}(t)=Q(D) x(t)=Q^{2}(D) x(t)=Q(D) x^{1}(t), \quad y^{1}(t)=(I-Q(D)) x(t)=(I-Q(D))^{2} x(t)=(I-Q(D)) y^{1}(t)$.

Таким образом, если $Q(D)$ дифферениируемо, то равенство (1) эквивалентно системе, состоящей из уравнений (7), (9), (10) в обозначениях (11) и равенства

$$
x(t)=x^{1}(t)+y^{1}(t)
$$

следующего из (9).

Замечание 1. Требование гладкости $Q(D)$ не означает гладкости $D(t)$, к примеру, если

$$
D(t)=\left(\begin{array}{c}
\sqrt{t} \\
2 \sqrt{t}
\end{array}\right)
$$

то

$$
Q(D)=\left(\begin{array}{rr}
0 & 0 \\
-2 & 1
\end{array}\right)
$$

Здесь $Q(D)$ гладкое отображение, хотя $D(t)$ не дифференцируемо при $t=0$.

Замечание 2. Можно прийти к уравнению (10) без использования проектора $Q(D)$, а некоторой заменой

$$
\left\{\begin{array}{l}
L_{1}(t) x(t)=x^{1}(t), \\
G_{1}(t) x(t)=y^{1}(t),
\end{array} \quad \operatorname{det}\left(\begin{array}{l}
L_{1}(t) \\
G_{1}(t)
\end{array}\right) \neq 0\right.
$$

предложенной в [4]. 
Функцию $x^{1}(t)$ называют функцией псевдосостояния (псевдосостоянием); функцию $y^{1}(t)$ называют функиией псевдоуправления (псевдоуправлением) первого шага декомпозиции.

Уравнение (10) аналогично исходному уравнению (1), только поиск решений должен осуществляться в более узком, чем исходное, пространстве, так как система (10) содержит меньшее количество скалярных уравнений, чем (1), за счет отщепления от (1) соотношений (7). Кроме того, условие $(2)$ переходит в условия и для $x^{1}(t)$, и для $y^{1}(t)$ :

$$
x^{1}\left(t_{i}\right)=Q\left(D\left(t_{i}\right)\right) x_{i}, \quad y^{1}\left(t_{i}\right)=\left(I-Q\left(D\left(t_{i}\right)\right)\right) x_{i} .
$$

Эти условия приводят с помощью равенства (10) к условию

$$
\dot{x}^{1}\left(t_{i}\right)=B_{1}\left(t_{i}\right) x^{1}\left(t_{i}\right)+D_{1}\left(t_{i}\right) y^{1}\left(t_{i}\right)+f_{1}\left(t_{i}\right) .
$$

Теперь задача состоит в построении $x^{1}(t)$ и $y^{1}(t)$, удовлетворяющих уравнению (10) и условиям

$$
\begin{aligned}
& x^{1}\left(t_{i}\right)=Q\left(D\left(t_{i}\right)\right) x_{i}=: x_{i}^{10}, \\
& \dot{x}^{1}\left(t_{i}\right)=B_{1}\left(t_{i}\right) x^{1}\left(t_{i}\right)+D_{1}\left(t_{i}\right) y^{1}\left(t_{i}\right)+f_{1}\left(t_{i}\right)=: x_{i}^{11},
\end{aligned}
$$

следующим из (2), (9) и (10) (=: означает «обозначим», второй индекс вверху - порядок производной вектор-функции).

Лемма 2 (см. [3]). Пусть $Q(D)$ дифферениируем на $\left[t_{0}, t_{k}\right]$. Уравнение (1) с условием (2) эквивалентно системе, состоящей из уравнений (7), (9), (10) с условиями (14).

Если в уравнении $(10)$ матрица $D_{1}(t)$ является сюръективной, то процесс декомпозиции на этом заканчивается, и следует приступить к построению $x^{1}(t)$ и $y^{1}(t)$, удовлетворяющих уравнению (10) и условиям (14). Способ построения описан ниже на последнем шаге декомпозиции.

Примером динамической системы с сюръективной матрицей $D_{1}(t)$ является система управления, приведенная в [7], описывающая движение в вертикальной плоскости материальной точки под действием реактивной силы. В этом примере уравнение (1) - это система

$$
\dot{x}_{1}(t)=x_{2}(t), \quad \dot{x}_{2}(t)=u_{1}(t), \quad \dot{x}_{3}(t)=x_{4}(t), \quad \dot{x}_{4}(t)=u_{2}(t)-g
$$

(описание параметров системы приведено ниже в примере (1)). Уравнение (7) - это система

$$
u_{1}(t)=\dot{x}_{2}(t), \quad u_{2}(t)=\dot{x}_{4}(t)+g .
$$

Уравнение (10) - это система

$$
\dot{x}_{1}(t)=x_{2}(t), \quad \dot{x}_{3}(t)=x_{4}(t) .
$$

Здесь

$$
x^{1}=\left(\begin{array}{l}
x_{1} \\
x_{3}
\end{array}\right), \quad y^{1}=\left(\begin{array}{l}
x_{2} \\
x_{4}
\end{array}\right), \quad B_{1}=\left(\begin{array}{ll}
0 & 0 \\
0 & 0
\end{array}\right), \quad D_{1}=\left(\begin{array}{ll}
1 & 0 \\
0 & 1
\end{array}\right) .
$$

Матрица $D_{1}$ не только сюръективная, но и обратимая за счет отсутствия у нее и коядра, и ядра.

3.2. Второй шаг декомпозиции. В случае $Q(D) \neq 0$ уравнение (10) относительно двух неизвестных вектор-функций $x^{1}(t)$ и $y^{1}(t)$ записывается в виде

$$
D_{1}(t) y^{1}(t)=\dot{x}^{1}(t)-B_{1}(t) x^{1}(t)-f_{1}(t) .
$$

Отображение $D_{1}(t)$ действует из $\operatorname{Im} D$ в Coker $D$. Для доказательства этого факта достаточно продифференцировать равенство

$$
Q(D)(I-Q(D))=0
$$

в результате чего получим

$$
\dot{Q}(D)(I-Q(D))=Q(D) \dot{Q}(D)
$$

тогда

$$
\begin{gathered}
\dot{Q}(D)(I-Q(D))=\dot{Q}(D)(I-Q(D))^{2}=Q(D) \dot{Q}(D)(I-Q(D)), \\
D_{1}(t)=Q(D)(\dot{Q}(D)+Q(D) B(t))(I-Q(D))
\end{gathered}
$$

(напомним, $Q(D)$ - проектор на Coker $D,(I-Q(D))$ - проектор на $\operatorname{Im} D)$. 
Во-вторых, из дифференцируемости $Q(D)$ следует, что $\operatorname{dim}$ Coker $D(t)=$ const (cм. [3]), т.е. отображение $D_{1}(t)$ при всех $t \in\left[t_{0}, t_{k}\right]$ действует из конечномерного подпространства в конечномерное подпространство, размерности которых постоянны, следовательно, его свойства аналогичны свойствам $D(t)$.

Введем обозначения $\operatorname{dim}$ Coker $D=n_{1}$ и $\operatorname{dim}$ Coker $D_{1}=n_{2}$. Имеем $n \geqslant n_{1} \geqslant n_{2}$.

Случай $n=n_{1}$ означает $\operatorname{Im} D(t)=\{0\}, D(t) \equiv 0$. В этом случае уравнение (1) имеет вид $\dot{x}=B(t) x$, и его решение $x(t)$ не может, вообще говоря, удовлетворить условиям (2), так как $k>0$. В этом случае исходная система не является полностью управляемой.

Случай $n_{1}=n_{2}$ означает, что $D_{1}(t) \equiv 0$, и решение $x^{1}(t)$ уравнения $\dot{x}^{1}=B_{1}(t) x^{1}$ (см. (10)) не может, вообще говоря, удовлетворить условиям (14), что противоречит полной управляемости исходной системы. Поэтому рассматриваются $n>n_{1}>n_{2}>\ldots$

Второй шаг использует тот факт, что уравнение (10) аналогично уравнению (1). С помощью $D_{1}(t)$ производится расщепление

$$
\operatorname{Im} D(t)=\operatorname{Coim} D_{1}(t) \dot{+} \operatorname{Ker} D_{1}(t), \quad \text { Coker } D(t)=\operatorname{Im} D_{1}(t) \dot{+} \text { Coker } D_{1}(t),
$$

и переход от уравнения (10) к уравнениям в подпространствах $\operatorname{Im} D_{1}$ и Coker $D_{1}$ и т. д.

3.3. Следующие декомпозииии. Введем следующие обозначения (при условии дифференцируемости соответствующих проекторов и $\left.n>n_{1}>n_{2}>\ldots>n_{i}\right)$ :

$$
\begin{gathered}
B_{0}(t)=B(t), \quad D_{0}(t)=D(t), \quad x^{0}(t)=x(t), \quad y^{0}(t)=u(t), \\
B_{j}(t)=\left(Q\left(D_{j-1}\right) B_{j-1}(t)+\dot{Q}\left(D_{j-1}\right)\right) Q\left(D_{j-1}\right), \\
D_{j}(t)=\left(Q\left(D_{j-1}\right) B_{j-1}(t)+\dot{Q}\left(D_{j-1}\right)\right)\left(I-Q\left(D_{j-1}\right)\right), \\
x^{j}(t)=Q\left(D_{j-1}\right) x^{j-1}(t), \quad y^{j}(t)=\left(I-Q\left(D_{j-1}\right)\right) x^{j-1}(t),
\end{gathered}
$$

$Q\left(D_{j-1}\right)$ и $P\left(D_{j-1}\right)$ - проекторы на Coker $D_{j-1}(t)$ и $\operatorname{Ker} D_{j-1}(t)$, соответственно, отвечающие разложениям

$$
\begin{gathered}
\operatorname{Im} D_{j-2}(t)=\operatorname{Coim} D_{j-1}(t) \dot{+} \operatorname{Ker} D_{j-1}(t), \\
\text { Coker } D_{j-2}(t)=\operatorname{Im} D_{j-1}(t) \dot{+} \operatorname{Coker} D_{j-1}(t) ; \\
n_{j}=\operatorname{dim} \text { Coker } D_{j-1}(t), \quad j=1,2, \ldots
\end{gathered}
$$

Неравенство $n>n_{1}>\ldots>n_{j}$ может иметь лишь два исхода: $n>n_{1}>\ldots>n_{p}=n_{p+1} \neq 0$ или $n>n_{1}>\ldots>n_{p}>n_{p+1}=0$. В первом случае $D_{p}(t) \equiv 0$, во втором $Q\left(D_{p}\right) \equiv 0$. Поскольку система (1) полностью управляемая, то $Q\left(D_{p}\right) \equiv 0$.

Таким образом, справедливо следующее утверждение.

Лемма 3. При дифферениируемости $Q\left(D_{j}\right), j=0,1, \ldots$ существует такое $p \in \mathbb{N}$, что уравнение (1) эквивалентно системе

$$
\begin{gathered}
u(t)=D^{-}(t)(\dot{x}(t)-B(t) x(t))+z(t), \\
x(t)=x^{1}(t)+y^{1}(t), \\
y^{j}(t)=D_{j}^{-}(t)\left(\dot{x}^{j}(t)-B_{j}(t) x^{j}(t)\right)+z^{j}(t), \\
x^{j}(t)=x^{j+1}(t)+y^{j+1}(t), \\
\dot{x}^{p}(t)=B_{p}(t) x^{p}(t)+D_{p}(t) y^{p}(t),
\end{gathered}
$$

для всех $z(t) \in \operatorname{Ker} D(t)$, всех $z^{j}(t)=P\left(D_{j}\right) y^{j}(t) \in \operatorname{Ker} D_{j}(t), j=\overline{1, p-1}$, где $D_{p}(t)$ сюрвективно.

Соотношения (16)-(20) получаются последовательным расщеплением, описанным выше, с использованием обозначений (15).

При выполнении условий леммы 3 имеем: Coker $D_{p-1}=\operatorname{Im} D_{p}$ и

$$
\mathbb{R}^{n}=\operatorname{Im} D \dot{+} \operatorname{Im} D_{1} \dot{+} \ldots \dot{+} \operatorname{Im} D_{p-1} \dot{+} \operatorname{Im} D_{p} .
$$


При этом

$$
\begin{aligned}
x(t)=x^{1}(t)+y^{1}(t)=x^{2}(t) & +y^{2}(t)+y^{1}(t)=\ldots= \\
& =\ldots=x^{p}(t)+\sum_{j=1}^{p} y^{j}(t)=x^{p}(t)+\sum_{j=1}^{p}\left(I-P\left(D_{j}\right)\right) x^{j}(t) .
\end{aligned}
$$

$\mathrm{B}$ частном случае стационарной системы управления схожие разложения пространства $\mathbb{R}^{n}$ и вектор-функции $x(t)$ получены другими методами в [8].

3.4. O краевых условиях. В (14) получены условия для $x^{1}(t)$ и $\dot{x}^{1}(t)$. Из $(15)$ находим условия для $x^{j+1}(t)$ :

$$
x^{j+1}\left(t_{i}\right)=x_{i}^{j+1,0}
$$

со значениями

$$
x_{i}^{j+1,0}=\left.Q\left(D_{j}\right)\right|_{t_{i}} x^{j}\left(t_{i}\right)=\left.\prod_{s=1}^{j+1} Q\left(D_{j-s+1}\right)\right|_{t_{i}} x_{i}^{00}, \quad i=\overline{0, k} .
$$

Из соотношения

$$
\dot{x}^{j+1}(t)=\left(\dot{Q}\left(D_{j}\right)+Q\left(D_{j}\right) B_{j}(t)\right) x^{j}(t)
$$

следует

$$
\left.\frac{d^{s} x^{j+1}}{d t^{s}}\right|_{t_{i}}=x_{i}^{j+1 s}
$$

где

$$
x_{i}^{j+1 s}=\left.\left(\frac{d^{s-1}}{d t^{s-1}}\left(\dot{Q}\left(D_{j}\right)+Q\left(D_{j}\right) B_{j}(t)\right) x^{j}(t)\right)\right|_{t_{i}}, \quad i=\overline{0, k}, \quad s=\overline{1, j+1} .
$$

Поскольку

$$
z^{j+1}(t)=P\left(D_{j+1}\right) y^{j+1}(t)=P\left(D_{j+1}\right)\left(I-Q\left(D_{j}\right)\right) x^{j}(t),
$$

то

$$
\left.\frac{d^{s} z^{j+1}(t)}{d t^{s}}\right|_{t_{i}}=z_{i}^{j+1 s}, \quad s=\overline{0, j}
$$

при

$$
z_{i}^{j+1 s}=\left.\frac{d^{s}}{d t^{s}} P\left(D_{j+1}\right)\left(I-Q\left(D_{j}\right)\right) x^{j}(t)\right|_{t_{i}} .
$$

3.5. Об эквивалентности задач.

Теорема 1 (см. [3]). Пусть $Q\left(D_{j}\right)$ и $f_{j}(t)$ дифферениируемы на $\left[t_{0}, t_{k}\right]$, матрицы

$$
\dot{Q}\left(D_{J}\right)+Q\left(D_{j}\right) B_{j}(t), \quad P\left(D_{j+1}\right)\left(I-Q\left(D_{j}\right)\right)
$$

дифферениируемы $j$ раз в точках $t_{i}, i=\overline{0, k}$. Тогда уравнение (1) с условиями (2) эквивалентно системе (16)-(20) с условиями (23), (24) и произвольными функииями $z_{j+1}(t) \in \operatorname{Ker} D_{j+1}(t)$, удовлетворяющими условиям (25), $j=\overline{0, p-1}$.

3.6. Построение псевдоуправления и псевдосостояния последнего шага декомпозииии. Выше осуществлен многошаговый переход от исходного уравнения (1) к уравнению (20):

$$
\dot{x}^{p}=B_{p}(t) x^{p}(t)+D_{p}(t) y^{p}(t)
$$

и от условий (2) к условиям, вытекающими из (23), (24):

$$
\left.\frac{d^{s} x^{p}}{d t^{s}}\right|_{t_{i}}=x_{i}^{p s}, \quad s=\overline{0, p}, i=\overline{0, k}
$$

При этом $p \in \mathbb{N}$ таково, что $D_{p}(t)$ - сюръекция, т.е. $Q\left(D_{p}\right) \equiv 0$. 
В качестве $x^{p}(t)$ можно взять линейную комбинацию линейно независимых функций $\varphi_{j}(t) \in \mathbb{R}^{1}$ с коэффициентами $\alpha_{j} \in$ Coker $D_{p-1}$, удовлетворяющими условиям (27):

$$
x^{p}(t)=\sum_{j=1}^{(p+1)(k+1)} \alpha_{j} \varphi_{j}(t), \quad \alpha_{j}=\left(\alpha_{j 1}, \alpha_{j 2}, \ldots, \alpha_{j \tau}\right), \quad \tau=\operatorname{dim} \operatorname{Im} D_{p}(t) .
$$

После подстановки этого выражения в условия (27) для каждого $\alpha_{j m}, m=1,2, \ldots, \tau$, образуется алгебраическая система с главным определителем $\Delta$. Первые $p+1$ строк этого определителя есть строки определителя Вронского для функций $\varphi_{j}(t), j=1,2, \ldots,(p+1)(k+1)$, в точке $t=0$. Следующие $p+1$ строк - строки определителя Вронского для тех же функций в точке $t_{1}, \ldots$, последние $p+1$ строк - строки определителя Вронского для $\varphi_{j}(t)$ в точке $t_{k}$. Если такой определитель отличен от нуля, то коэффициенты $\alpha_{j}$ определяются однозначно. В частности, $\Delta \neq 0$, если $\varphi_{j}(t)=t^{j}$ (см. [6]).

Далее из соотношения (18) с $j=p$ находим $y^{p}(t)$ :

$$
y^{p}(t)=D_{p}^{-}(t)\left(\dot{x}^{p}-B_{p}(t) x^{p}(t)\right)+z^{p}(t)
$$

для любого $z^{p}(t) \in \operatorname{Ker} D_{p}(t)$, удовлетворяющего условиям (25) при $j=p-1$. Поскольку в качестве $x^{p}(t)$ и $z^{p}(t)$ можно выбрать сколь угодно гладкие вектор-функции, то гладкость $y^{p}(t)$ зависит от гладкости коэффициентов $D_{p}^{-}(t)$ и $B_{p}(t)$.

3.7. Построение функций состояния и управления задачи (1), (2). Функции $x^{p}(t)$ и $y^{p}(t)$, построенные в предыдущем пункте, в сумме образуют функцию $x^{p-1}(t)$ (формула $(19)$ с $j=p-1$ ). Затем находим $y^{p-1}$ по формуле (18) с $j=p-2$. Эти две последние функции образуют в сумме функцию $x^{p-2}$ (формула (19) с $j=p-2$ ), и т. д. Формула (17) определяет функцию $x(t)$, а формула $(16)$ - функцию $u(t)$. От функций $z^{j}(t)=P\left(D_{j}\right) y^{j}(t)$ требуется лишь достаточная гладкость и подчинение условиям (25).

При каскадном расщеплении исходного уравнения (1) на уравнения в подпространствах на каждом этапе требовалась дифференцируемость по $t$ проекторов $Q\left(D_{j}\right), j=\overline{0, p-1}$.

Для выведения многоточечных условий для функции $x^{p}(t)$ требуется существование производных в точках $t_{i}, i=\overline{0, k}$, от выражений $\dot{Q}\left(D_{j}\right)+Q\left(D_{j}\right) B_{j}(t)$ и $P\left(D_{j+1}\right)\left(I-Q\left(D_{j}\right)\right)$ до порядкај, $j=\overline{0, p-1}$.

Для возможности пользоваться формулами (18) требуется существования производных от функций $x^{j}(t), j=\overline{0, p-1}$.

Если в исходной задаче управления требуется предъявить непрерывную управляющую функцию $u(t)$, то для этого требуется выполнение условий $Q\left(D_{j}\right) \in C^{1}\left(\left[t_{0}, t_{k}\right] \rightarrow\right.$ Coker $\left.D_{j}\right), B_{j}(t) \in$ $C^{j}\left(\left[t_{0}, t_{k}\right] \rightarrow \operatorname{Coker} D_{j-1}\right)$ и $D_{j}^{-}(t) \in C^{j}\left(\left[t_{0}, t_{k}\right] \rightarrow \operatorname{Coim} D_{j}\right), j=\overline{0, p-1}$.

Полученные результаты приводят к следующей теореме.

Теорема 2. Пусть матрицы $B(t)$ и $D(t)$ обладают следующими свойствами:

(1) $Q\left(D_{j}\right) \in C^{1}\left(\left[t_{0}, t_{k}\right] \rightarrow\right.$ Coker $\left.D_{j}\right)$;

(2) $B_{j}(t) \in C^{j}\left(\left[t_{0}, t_{k}\right] \rightarrow\right.$ Coker $\left.D_{j-1}\right), D_{j}^{-}(t) \in C^{j}\left(\left[t_{0}, t_{k}\right] \rightarrow \operatorname{Coim} D_{j}\right)$;

(3) матрицы $\dot{Q}\left(D_{j}\right)+Q\left(D_{j}\right) B_{j}(t)$ и $P\left(D_{j+1}\right)\left(I-Q\left(D_{j}\right)\right)$ дифференцируемъ в точках $t_{i}, i=\overline{0, k}$, до порядка $j$

(4) $f_{j}(t) \in C^{j}\left(\left[t_{0}, t_{k}\right] \rightarrow\right.$ Coker $\left.D_{j-1}\right), j=\overline{0, p}$.

Тогда существует непрерывная вектор-функиия $u(t) \in \mathbb{R}^{m}$, при подстановке которой в систему (1), решение $x(t)$ полученной системы удовлетворяет условиям (2).

При подстановке найденной таким образом управляющей функции $u(t)$ в $(1)$, решение $x(t)$ полученного уравнения совпадает с $x(t)$, найденным описанным выше способом. Действительно, решение задачи Коши

$$
\dot{x}(t)=B(t) x(t)+D(t) u(t), \quad x\left(t_{0}\right)=x_{0}^{00},
$$

единственно в силу непрерывности $B(t)$ и $D(t) u(t)$, следовательно, решением является именно функция $x(t)$, построенная выше. 
В случае постоянных матриц $B, D$ условия 1-3 теоремы 2 выполняются автоматически.

4. О решении задачи сохранения траектории при возмущениях специального вида. Применение леммы к уравнению (3) дает систему

$$
\begin{gathered}
Q(D)\left(I+H_{1}(t)\right) \dot{x}_{v}(t)=Q(D)\left(\left(B(t)+H_{2}(t)\right) x_{v}(t)+H_{3}(t) u_{v}(t)+f(t)+h(t)\right), \\
\left(I+D^{-}(t) H_{3}(t)\right) u_{v}(t)=D^{-}(t)\left(\left(I+H_{1}(t)\right) \dot{x}_{v}(t)-\left(B(t)+H_{2}(t)\right) x_{v}(t)-f(t)-h(t)\right)+z_{v}(t),
\end{gathered}
$$

для всех $z_{v}(t) \in \operatorname{Ker} D(t)$. Пусть выполняются следующие условия:

(i) $Q(D) H_{i}(t) \equiv 0, i=1, \ldots, 3 ; Q(D) h(t) \equiv 0, t \in\left[t_{0}, t_{k}\right]$;

(ii) $\left\|D^{-}(t) H_{3}(t)\right\|<1$.

Условие (i) означает, что $H_{i}(t)$ действуют в $\operatorname{Im} D(t)$ и $h(t) \in \operatorname{Im} D(t)$ (возмущения специального вида). Тогда (30) приобретает вид уравнения

$$
Q(D) \dot{x}_{v}(t)=Q(D)\left(B(t) x_{v}(t)+f(t)\right),
$$

которое не отличается от уравнения (8) для $x(t)$. Условия (2) одинаковые для $x(t)$ и $x_{v}(t)$, поэтому из уравнений $(8)$ и $(32)$ и условий $(2)$ можно найти одинаковые решения $x(t)$ и $x_{v}(t)$. Тогда из $(31)$

$$
u_{v}(t)=\left(I+D^{-}(t) H_{3}(t)\right)^{-1}\left(D^{-}(t)\left(\left(I+H_{1}(t)\right) \dot{x}(t)-\left(B(t)+H_{2}(t)\right) x(t)-f(t)-h(t)\right)+z_{v}(t)\right)
$$

для всех $z_{v}(t) \in \operatorname{Ker} D(t)$. Добавки $z_{v}(t)$ и $z(t)$ в формулах (33) и (7) не влияют на величину вектор-функций состояний $x_{v}(t)$ и $x(t)$, поэтому при решении задач инвариантности их можно взять одинаковыми.

Использование свойства

$$
\left(I+D^{-}(t) H_{3}(t)\right)^{-1}=I-D^{-}(t) H_{3}(t)\left(I+D^{-}(t) H_{3}(t)\right)^{-1}
$$

и (7) приводят к результату

$$
\begin{aligned}
& u_{v}(t)-u(t)=\left(I+D^{-}(t) H_{3}(t)\right)^{-1} D^{-}(t)\left(H_{1}(t) \dot{x}(t)-H_{2}(t) x(t)-h(t)\right)- \\
&-D^{-}(t) H_{3}(t)\left(I+D^{-}(t) H_{3}(t)\right)^{-1} D^{-}(t)(\dot{x}(t)-B(t) x(t)-f(t)),
\end{aligned}
$$

откуда видно, что если помехи $H_{i}(t)$ и $h(t)$ малы, то $u_{v}(t)$ мало отличается от $u(t)$, рассчитанного по формуле (7).

5. Примеры динамических систем с возмущениями специального вида. В предыдущем пункте рассмотрены возмущающие элементы в уравнении (3) со свойствами

$$
Q(D) H_{i}(t) \equiv 0, \quad i=1, \ldots, 3, \quad Q(D) h(t) \equiv 0, \quad t \in\left[t_{0}, t_{k}\right]
$$

(свойства (i)). Такими свойствами могут обладать, например, возмущающие элементы в линейных математических моделях, полученных на основе законов динамики Ньютона. В этом случае $a(t)=\ddot{x}(t)$, где $a(t)$ - ускорение движения $x(t)$, возникающего в результате действия некоторой силы, $x(t)=\left(x_{1}(t), \ldots, x_{n}(t)\right), n$-размерность пространства, в котором решается задача. Уравнение, описывающее движение, проектируется на координатные оси и в полученных соотношениях делаются замены $\dot{x}_{i}(t)=x_{i+1}(t), i=1,2, \ldots, n-1$. В последние равенства, как правило, возмущающие факторы не включаются, в результате чего в полученной математической модели возмущающие параметры оказываются в тех же скалярных уравнениях, что и компоненты управляющих вектор-функций. Может оказаться, что $H_{i}(t)$ действуют в $\operatorname{Im} D(t), h(t) \in \operatorname{Im} D(t)$, что иллюстрирует следующий пример.

Пример 1. Уравнение Мещерского (см. [7]), описывающее движение материальной точки массы $m=m(t)$, движущейся в вертикальной плоскости $\{\xi, \eta\}$ в поле силы тяжести под действием реактивной силы $f=f(t)$, возникающей в результате отделения от нее частиц с элементарной массой $d m_{1}$, имеет вид

$$
m \dot{v}=(s-v) \dot{m}_{1}+p
$$


где $m=m(t)=m_{0}+m_{1}(t) ; m_{0}$ - постоянная часть массы точки; $m_{1}=m_{1}(t)$ - реактивная масса точки; $v=v(t)$ - вектор абсолютной скорости точки; $s=s(t)$ - вектор скорости частицы; $p-$ вес. Равенство (34) проектируется на горизонтальную и вертикальную координатные оси:

$$
m \ddot{\xi}=(s-v)_{\xi} \dot{m}_{1}, \quad m \ddot{\eta}=(s-v)_{\eta} \dot{m}_{1}+p,
$$

$g \approx 9,8 \mathrm{~m} / \mathrm{c}^{2}$ (в системе единиц СИ). В [7] введены обозначения $x_{1}=\xi, x_{2}=\dot{\xi}, x_{3}=\eta, x_{4}=\dot{\eta}$, $u_{1}=(s-v)_{\xi} \dot{m}_{1} / m, u_{2}=(s-v)_{\eta} \dot{m}_{1} / m$. Функции $u_{1}=u_{1}(t), u_{2}=u_{2}(t)$ - компоненты управляющей вектор-функции в невозмущенной системе, полученной с помощью введенных обозначений из уравнений (35):

$$
\dot{x}_{1}=x_{2}, \quad \dot{x}_{2}=u_{1}, \quad \dot{x}_{3}=x_{4}, \quad \dot{x}_{4}=u_{2}-g .
$$

Пусть изменилась масса $m_{0}$ на величину $m_{2}=$ const, соответственно изменился вес на величину $m_{2} g$ и абсолютная скорость точки на величину $v_{1}=v_{1}(t)=\left(v_{1 \xi}, v_{1 \eta}\right)$. Пусть, кроме того, на материальную точку действует дополнительная внешняя сила $\varphi=\varphi(t)=\left(\varphi_{\xi}, \varphi_{\eta}\right)$. Уравнение Мещерского принимает вид

$$
m_{v} \dot{v}_{v}=\left(s-v_{v}\right) \dot{m}_{1}+p_{v}+\varphi
$$

где $m_{v}=m+m_{2}, v_{v}=v+v_{1}, p_{v}=p+m_{2} g$. Оно проектируется на координатные оси:

$$
m_{v} \ddot{\xi}_{v}=\left(s-v_{v}\right)_{\xi} \dot{m}_{1}+\varphi_{\xi}, \quad m_{v} \ddot{\eta}_{v}=\left(s-v_{v}\right)_{\eta} \dot{m}_{1}+p_{v}+\varphi_{\eta} .
$$

Возмущенная система (37) в обозначениях

$$
x_{v 1}=\xi_{v}, \quad x_{v 2}=\dot{\xi}_{v}, \quad x_{v 3}=\eta_{v}, \quad x_{v 4}=\dot{\eta_{v}}, \quad u_{v 1}=\left(s-v_{v}\right)_{\xi} \frac{\dot{m}_{1}}{m_{v}}, \quad u_{v 2}=\left(s-v_{v}\right)_{\eta} \frac{\dot{m}_{1}}{m_{v}}
$$

имеет вид

$$
\dot{x}_{v 1}=x_{v 2}, \quad \dot{x}_{v 2}=u_{v 1}+\frac{\varphi_{\xi}}{m_{v}}, \quad \dot{x}_{v 3}=x_{v 4}, \quad \dot{x}_{v 4}=u_{v 2}-g+\frac{\varphi_{\eta}}{m_{v}},
$$

с компонентами $u_{v 1}, u_{v 2}$ управляющей вектор-функции.

Каскадный метод, описанный выше, решения многоточечных задач для систем (36) и (38) предполагает нахождение компонентов движения из первого и третьего уравнений систем (36) и (38) и заданных многоточечных условий (см. п. 3.1). При этом эти уравнения одинаковы для невозмущенной и возмущенной систем (36) и (38), следовательно, их решения $x(t)$ и $x_{v}(t)$ одинаковы при реализованном начальном состоянии $x\left(t_{0}\right)=x_{v}\left(t_{0}\right)$ в силу единственности решения начальных задач для соответствующих систем этих уравнений. Используя равенство $x_{v}(t) \equiv x(t), t \in\left[t_{0}, t_{k}\right]$, и формулы для нахождения $u_{1}, u_{2}, u_{v 1}$ и $u_{v 2}$ из вторых и четвертых уравнений систем $(36)$ и $(38)$ получаем

$$
u_{v 1}=u_{1}-\frac{\varphi_{\xi}}{m_{v}}, \quad u_{v 2}=u_{2}-\frac{\varphi_{\eta}}{m_{v}}
$$

Из соотношений $(39)$ видно, что если $|\varphi|$ и $m_{2}$ малы, то и разности $u_{1 v}-u_{1}$ и $u_{2 v}-u_{2}$ малы, т.е. небольшое изменение управления позволяет сохранить программную траекторию движения материальной точки.

В [7] допущено, что абсолютная величина вектора $s-v$ задана и равна $\sigma$; тогда

$$
u_{1}=\sigma \cos \alpha_{\xi} \frac{\dot{m}_{1}}{m}, \quad u_{2}=\sigma \cos \alpha_{\eta} \frac{\dot{m}_{1}}{m},
$$

где $\alpha_{\xi}, \alpha_{\eta}$ - углы, составленные вектором $s-v$ с осями $\xi$ и $\eta$. Можно, следуя [7], допустить, что абсолютная величина скорости вектора $s-v_{v}$ задана и равна $\delta$, и ввести обозначение

$$
u_{v 1}=\delta \cdot \cos \beta_{\xi} \cdot \frac{\dot{m}_{v}}{m_{v}}, \quad u_{v 2}=\delta \cdot \cos \beta_{\eta} \cdot \frac{\dot{m}_{v}}{m_{v}},
$$

где $\beta_{\xi}$ и $\beta_{\eta}$ - углы между вектором $s-v-v_{1}$ и осями $\xi$ и $\eta ;\left(\dot{m}_{v}=\dot{m}=\dot{m}_{1}\right)$.

Соотношение (39) и указанные обозначения позволяют установить связь между направляющими косинусами:

$$
\delta \cos \beta_{\xi} \frac{\dot{m}}{m_{v}}=\sigma \cos \alpha_{\xi} \frac{\dot{m}_{1}}{m}-\frac{\varphi_{\xi}}{m_{v}}, \quad \delta \cos \beta_{\eta} \frac{\dot{m}}{m_{v}}=\sigma \cos \alpha_{\eta} \frac{\dot{m}}{m}-\frac{\varphi_{\eta}}{m_{v}}
$$


или

$$
\cos \beta_{\xi}=\frac{\sigma}{\delta} \frac{m_{v}}{m} \cos \alpha_{\xi}-\frac{\varphi_{\xi}}{\delta \dot{m}_{1}}, \quad \cos \beta_{\eta}=\frac{\sigma}{\delta} \frac{m_{v}}{m} \cos \alpha_{\eta}-\frac{\varphi_{\eta}}{\delta \dot{m}_{1}} .
$$

Свойством (i) обладают также возмущающие элементы в линеаризованных динамических системах управления движением спутника по экваториальной круговой орбите на постоянной высоте над поверхностью Земли (см. [1]); движением спутника связи, вращающегося за Луной вокруг точки равновесия (центра масс) системы Земля-Солнце-Луна для изучения обратной стороны Луны (см. [1]).

Однако не все системы управления, основанные на втором законе Ньютона, обладают свойством (i), что подтверждает следующий пример.

Пример 2. В [1] приведен пример системы, описывающей движение вертолета, для которого управляемой переменной является угол наклона $\theta$, изменяемый за счет углового положения несущего винта $\delta$ :

$$
\left\{\begin{array}{l}
\ddot{x}(t)=g \theta(t)-a_{1} \dot{\theta}(t)-b_{1} \dot{x}(t)+g \delta(t), \\
\ddot{\theta}(t)=-b_{2} \dot{\theta}(t)-a_{2} \dot{x}(t)+n \delta(t)
\end{array}\right.
$$

где $x$ - перемещение вертолета в горизонтальном направлении. Для вертолета боевого назначения приведены значения параметров $a_{1}, a_{2}, b_{1}, b_{2}$. С введением обозначений $x_{1}=x, x_{2}=\dot{x}, x_{3}=\theta$, $x_{4}=\dot{\theta}$ система (40) принимает вид

$$
\left\{\begin{array}{l}
\dot{x}_{1}=x_{2} \\
\dot{x}_{2}=-b_{1} x_{2}+g x_{3}-a_{1} x_{4}+g \delta \\
\dot{x}_{3}=x_{4} \\
\dot{x}_{4}=-a_{2} x_{2}-b_{2} x_{4}+n \delta
\end{array}\right.
$$

Если в системе (40) появляются возмущающие параметры, то они появляются в тех же уравнениях системы (41), где находится управляющая переменная $\delta_{v}$. Если, например, на вертолет действует некоторая внешняя сила $h=h(t)$, то система $(41)$ принимает вид

$$
\left\{\begin{array}{l}
\dot{x}_{v 1}=x_{v 2}, \\
\dot{x}_{v 2}=-b_{1} x_{v 2}+g x_{v 3}-a_{1} x_{v 4}+g \delta_{v}+h_{1}, \\
\dot{x}_{v 3}=x_{v 4} \\
\dot{x}_{v 4}=-a_{2} x_{v 2}-b_{2} x_{v 4}+n \delta_{v}+h_{2} .
\end{array}\right.
$$

Вектор $\left(0, h_{1}, 0, h_{2}\right)$ принадлежит $\operatorname{Im} D$ лишь если $n h_{1}=g h_{2}$, поскольку множество значений оператора $D=(0, g, 0, n)^{T}$ 一это $\{(0, g c, 0, n c)\}$ для всех $c \in \mathbb{R}^{1}$.

6. О блокировке возмущений общего вида. Если возмущающие элементы в динамической системе не обладают свойством (i), то можно, если это позволяет динамическая система, поставить перед возмущающими элементами блокатор $D(t)$. Если размерность $m$ управляющей вектор-функции меньше размерности $n$ исходного пространства, то следует вектор $u_{v}(t)$ и матрицу $D(t)$ дополнить нулевыми компонентами так, чтобы $u_{v}(t) \in \mathbb{R}^{n}$ и $D(t): n \times n$. В результате возмущенная система принимает вид

$$
\left(I+D(t) H_{1}(t)\right) \dot{x}_{v}(t)=\left(B(t)+D(t) H_{2}(t)\right) x_{v}(t)+\left(D(t)+D(t) H_{3}(t)\right) u_{v}(t)+f(t)+D(t) h(t) .
$$

Теперь $D(t) H_{i}(t), i=1, \ldots, 3$, действуют в $\operatorname{Im} D(t), D(t) h(t) \in \operatorname{Im} D(t)$. Пусть

(iii) $\left\|H_{3}(t)\right\|<1$.

Система (43) эквивалентна в силу леммы и условия (iii) системе

$$
\begin{gathered}
u_{v}(t)=\left(I+H_{3}(t)\right)^{-1} D^{-}(t)\left(\left(I+D(t) H_{1}(t)\right) \dot{x}_{v}(t)-\right. \\
\left.-\left(B(t)+D(t) H_{2}(t)\right) x_{v}(t)-f(t)-D(t) h(t)\right)+z_{v}(t) \quad \forall z_{v}(t) \in \operatorname{Ker} D(t), \\
Q(D) \dot{x}_{v}(t)=Q(D)\left(B(t) x_{v}(t)+f(t)\right)
\end{gathered}
$$


Соотношение (44) есть формула для нахождения $u_{v}(t)$ как функции от $x_{v}(t)$, а $x_{v}(t)$ находится из уравнения (45) с помощью многоточечных условий. Уравнение (45) не отличается от уравнения (8) для нахождения $x(t)$, поэтому $x_{v}(t) \equiv x_{v}(t), t \in\left[t_{0}, t_{k}\right]$.

На основании идентичности уравнений $(8),(32)$ и (45) для нахождения $x(t)$ и $x_{v}(t)$ справедлива следующая теорема.

Теорема 3. При определенной гладкости $B(t), D(t), f(t)$ задачи (1), (2) и еqз, (2) или (43), (2) с непрерывными помехами $H_{i}(t), i=1, \ldots, 3, h(t)$, обладающими свойствами (i), (ii), или с помехами $D H_{i}(t)$, Dh(t) со свойством (iii) разрешимы или неразрешимы одновременно.

Очевидно, что условия на гладкость $B(t), D(t)$ и $f(t)$ для каскадной декомпозиции систем (3) и (43) те же, что и для декомпозиции системы (1) (см. теорему 2).

Замечание 3. Задача (1), (2) при достаточной гладкости $B(t), D(t), f(t)$ разрешима тогда и только тогда, когда система (1) является полностью управляемой (см. [2]).

Далее отметим, что $D^{-}(t) D(t)=(I-P(D))$, следовательно,

$$
\begin{gathered}
D^{-}(t) D(t) H_{i}(t)=(I-P(D)) H_{i}(t), \quad i=1, \ldots, 3 ; \\
D^{-}(t) D(t) h(t)=(I-P(D)) h(t),
\end{gathered}
$$

откуда следует, что «части» $P(D) H_{i}(t)$ и $P(D) h(t)$ в формуле $(44)$ не участвуют, следовательно, система (43) робастна по отношению к этим «частям» возмущений.

Из (44) и того факта, что $x_{v}(t) \equiv x(t)$, следует

$$
\begin{aligned}
u_{v}(t)=\left(I+H_{3}(t)\right)^{-1} & \left(D^{-}(t)(\dot{x}(t)-B(t) x(t)-f(t))+\right. \\
& \left.+(I-P(D))\left(H_{1}(t) \dot{x}(t)-H_{2}(t) x(t)-h(t)\right)\right)+z_{v}(t) \quad \forall z_{v}(t) \in \operatorname{Ker} D(t) .
\end{aligned}
$$

Как отмечено в п. 3, можно положить $z_{v}(t) \equiv z(t)$.

Отсюда и из (7) имеем

$$
\begin{aligned}
u_{v}(t)-u(t)=\left(I+H_{3}(t)\right)^{-1}(I-P(D))\left(H_{1}(t) \dot{x}(t)-H_{2}(t) x(t)-h(t)\right)- & \\
& -H_{3}(t)\left(I+H_{3}(t)\right)^{-1} D^{-}(t)(\dot{x}(t)-B(t) x(t)-f(t)),
\end{aligned}
$$

откуда видно, что при достаточно малых возмущениях $H_{i}(t), h(t)$ разность $u_{v}(t)-u(t)$ мала.

Таким образом, доказано следующее утверждение.

Теорема 4. Пусть $B(t), D(t), f(t)$ достаточно гладкие и $G_{i}(t), i=1, \ldots, 3, h(t)$ - непреръьны и удовлетворяют условиям (i), (ii) или (iii). При управляющем воздействии $u_{v}(t)$ формуль (33) или (44) состояния возмущенных систем (3) или (43) с условиями (2) не зависят от возмущений $G_{i}(t), i=1, \ldots, 3, h(t)$ и равны состоянию невозмущенной системы (1) с условиями (2).

7. Пример гашения возмущений. Вернемся к примеру 2. Первый шаг каскадного расщепления системы (41) состоит в получении формулы для нахождения $\delta$ из второго (поскольку $g \neq 0$ ) уравнения в (41):

$$
\delta=\frac{1}{g}\left(\dot{x}_{2}+b_{1} x_{2}-g x_{3}+a_{1} x_{4}\right)
$$

и условия корректности системы (41) вида (8). Для этого следует второе равенство в (41) умножить на $n$, четвертое на $g$ и вычесть из одного полученного соотношения другое. Условие корректности имеет вид

$$
\dot{x}_{1}=x_{2}, \quad \dot{x}_{3}=x_{4}, \quad n \dot{x}_{2}-g \dot{x}_{4}=\left(g a_{2}-n b_{1}\right) x_{2}+n g x_{3}+\left(g b_{2}-n a_{1}\right) x_{4} .
$$

Решение системы (47) с $n=n(t)$ с условиями (2) описано в [4]. 
В системе (41) следует записать $u_{v}$ и $D$, как рекомендовано в п. 5 , например в виде

$$
u_{v}=\left(\begin{array}{l}
\delta \\
0 \\
0 \\
0
\end{array}\right), \quad D=\left(\begin{array}{cccc}
0 & 0 & 0 & 0 \\
g & 0 & 0 & 0 \\
0 & 0 & 0 & 0 \\
n & 0 & 0 & 0
\end{array}\right)
$$

Введение в (42) блокатора $D$ перед возмущением $\left(0, h_{1}, 0, h_{2}\right)$ приводит систему $(42)$ к виду

$$
\left\{\begin{array}{l}
\dot{x}_{v 1}=x_{v 2}, \\
\dot{x}_{v 2}=-b_{1} x_{v 2}+g x_{v 3}-a_{1} x_{v 4}+g \delta_{v}+g h_{1}, \\
\dot{x}_{v 3}=x_{v 4}, \\
\dot{x}_{v 4}=-a_{2} x_{v 2}-b_{2} x_{v 4}+n \delta_{v}+n h_{1} .
\end{array}\right.
$$

Из второго уравнения этой системы определяется $\delta_{v}$ :

$$
\delta_{v}=\frac{1}{g}\left(\dot{x}_{v 2}+b_{1} x_{v 2}-g x_{v 3}+a_{1} x_{v 4}-g h_{1}\right) .
$$

Условие корректности системы $(48)$ - это

$$
\dot{x}_{v 1}=x_{v 2}, \quad \dot{x}_{v 3}=x_{v 4}, \quad n \dot{x}_{v 2}-g \dot{x}_{v 4}=\left(g a_{2}-n b_{1}\right) x_{v 2}+n g x_{v 3}+\left(g b_{2}-n a_{1}\right) x_{v 4} .
$$

Последняя система не отличается по виду от системы $(47)$ для нахождения $x=x(t)$, и поскольку $x_{v}(t)$, как и $x(t)$, удовлетворяет условиям $(2)$, то $x_{v}(t) \equiv x(t)$.

Разность $\delta_{v}(t)-\delta(t)$, как следует из (49) и $(46)$ :

$$
\delta_{v}(t)-\delta(t)=-h_{1}(t)
$$

мала, если мало $h_{1}(t)$, т.е. мало возмущение $\left(0, g h_{1}(t), 0, n h_{1}(t)\right)$.

8. Заключение. Рассмотрена линейная нестационарная полностью управляемая динамическая система. При достаточной гладкости параметров системы существует управление, переводящее состояние системы из произвольного начального в произвольное конечное состояние через любое конечное количество контрольных точек. При появлении в системе внешних и внутренних помех, шумов, изменений параметров системы. т.е. возмущений, решена задача построения нового управления такого, что состояние динамической системы не реагирует на возмущения, остается тем же, что и при отсутствии возмущений (инвариантность относительно возмущений).

Возмущения предполагаются известными, управление программное. Рассматриваются возмущения специального вида, а именно, действующие в подпространство, являющееся множеством значений операторного коэффициента, стоящего в уравнении при управляющей вектор-функции. В этом случае приведены формулы для определения управляющих вектор-функций невозмущенной и возмущенной систем, для получения которых используется метод каскадной декомпозиции поставленных задач.

Сравнение управляющих вектор-функций для невозмущенной и возмущенной систем показывает, что при малых возмущениях достаточно немного изменить управление, чтобы состояние системы не реагировало на возмущения.

Если же возмущающие факторы не являются возмущениями специального вида, описанного выше, то их можно погасить с помощью того же операторного коэффициента, стоящего в уравнении при управляющей вектор-функции, поставив его в системе перед каждым возмущением, если это позволяет реальная динамическая система. В этом случае также построена управляющая вектор-функция возмущенной системы, производится сравнение управляющих вектор-функций для одинаковых состояний невозмущенной и возмущенной систем.

\section{СПИСОК ЛИТЕРАТУРЫ}

1. Дорф Р., Бишоп Р. Современные системы управления. - М.: Лаборатория базовых знаний, 2002.

2. Зубова С. П. Решение обратных задач для линейных динамических систем каскадным методом// Докл. PAH. - 2012. - 447, № 6. - C. 599-602. 
3. Зубова С. П. Метод каскадной декомпозиции решения задач для псевдорегулярных уравнений/ Дисс. на соиск. уч. степ. доктора физ.-мат. наук - Воронеж, 2013.

4. Зубова С. П., Раецкая Е. В. Алгоритм решения линейных многоточечных задач управления методом каскадной декомпозиции// Автомат. телемех. - 2017. - 7. - С. 22-38.

5. Зубова С. П., Раецкая Е. В., Ле Хай Чунг О полиномиальных решениях линейной стационарной системы управления// Автомат. телемех. - 2008. - 11. - С. 41-47.

6. Каган В. Ф. Основания теории определителей. - Одесса, 1922.

7. Красовский Н. H. Теория управления движением. - М.: Наука, 1968.

8. Уонэм М. Линейные многомерные системы управления: геометрический подход. - М.: Наука, 1980.

Зубова Светлана Петровна

Воронежский государственный университет

E-mail: spzubova@.mail.ru

Раецкая Елена Владимировна

Воронежский государственный лесотехнический университет им. Г. Ф. Морозова

E-mail: raetskaya@inbox.ru

Ле Хай Чунг

Данангский научно-педагогический университет, Дананг, Вьетнам

E-mail: lhtrung@ued.udn.vn 